\title{
Clues to Dural Arteriovenous Fistulas in Patients with Progressive Dementia
}

\author{
Amir R. Dehdashti, Charles C. Matouk, Karel TerBrugge, M. Christopher Wallace, \\ Robert A. Willinsky
}

Can. J. Neurol. Sci. 2010; 37: 532-534

The mode of presentation of cranial dural arteriovenous fistulas (DAVFs) is dictated by the pattern of venous drainage. Lesions without demonstrable cortical venous reflux (CVR) have an indolent natural history. Alternatively, the presence of CVR defines aggressive lesions that mandate early diagnosis and definitive intervention (either surgical or endovascular). ${ }^{1}$ Indeed, it has been previously reported that untreated aggressive DAVF have an annual event rate of $15 \%$ and an annual mortality rate of $10.4 \% .^{2}$ While the majority of aggressive DAVFs present with a hemorrhagic complication, an under-recognized presentation is progressive cognitive impairment related to venous congestive encephalopathy. ${ }^{3,4}$ The largest series to date was reported by Hurst et al who summarized the clinical and radiographic features of five patients with DAVF presenting with dementialike syndromes. ${ }^{5}$ In this series, however, four of the five patients demonstrated a concomitant decreased level of consciousness or lateralizing findings at presentation. We therefore sought to identify clues on history, physical examination and MRI (magnetic resonance imaging) that would alert the clinician in the absence of decreased level of consciousness or lateralizing findings to a diagnosis of DAVF.

\section{Methods}

We retrospectively reviewed 246 consecutive patients with an angiographically confirmed cranial DAVF, investigated at the University of Toronto Brain Vascular Malformation Study Group. A relational database was maintained between June 1984 and May 2008, prospectively since 1989. Assessments were performed by a multi-disciplinary team comprising cerebrovascular neurosurgeons and interventional neuroradiologists. A detailed history, physical examination, magnetic resonance imaging before and after treatment, and conventional digital subtraction angiograms were documented for all patients. Periventricular hyperintensities most commonly seen with small vessel disease were not considered as abnormalities related to the DAVF.

Treatment was evaluated and patients' mini-mental state examination (MMSE) were assessed before and after treatment. This study was approved by the hospital Ethics Committee.

\section{RESULTS}

Eight of 246 patients (3\%) were identified whose primary mode of presentation was progressive cognitive deficits without a decreased level of consciousness or lateralizing findings. These included five women and three men with a median age of 62 years (range, 48 to 86 years). Cognitive deficits were characterized by prominent personality changes, impairment of short- and long-term memory, and decreased concentration. Symptoms were progressive and present for a median four weeks (range: 3 to 24 weeks) prior to diagnosis.

Associated symptoms were manifest in all patients that are not very typical in common dementia syndromes. These included worsening headache (four patients), gait instability (three patients), generalized weakness (one patient) and first-time seizure (one patient). Physical examination was remarkable in four patients for the presence of cranial bruit, in two for engorged and prominent scalp veins and in two for arm tremor.

Magnetic resonance imaging revealed engorged venous channels over the surface of the cerebral and/or cerebellar hemispheres in all patients. We did not identify any patient in this series with normal MRI and the DAVF/dementia association. Other pathological findings included hyperintensities on T2weighted images (five patients), hemorrhage (three patients) and ventriculomegaly (one patient). Review of the history in the three patients with hemorrhage indicated prior cognitive deficit, before the hemorrhagic event. Their MMSE was however assessed at the time of admission. None of the hemorrhage was life threatening with need for urgent intervention. Digital subtraction angiograms confirmed the diagnosis of DAVF and revealed the location of the fistula: transverse sinus (four patients, two had also torcular involvement, one with rectus sinus involvement), superior sagittal sinus (three patients) and anterior cranial fossa (one patient). Three patients had multiple DAVFs. Cortical venous reflux was invariably present. All patients underwent successful treatment by disconnection of the fistula (surgical in five cases and endovascular transarterial in the remaining) with regression of the dementia-like syndrome in five patients. The improvement was based on a significant increase in their MMSE score to more than 24 in those five patients. Magnetic resonance hyperintensities present in five cases of this series, improved in four after treatment. There was no complication related to surgical or endovascular treatment in any of these patients.

From the University of Toronto Brain Vascular Malformation Study Group, Division of Neurosurgery(ARD, CCM, MCW), Department of Medical Imaging (KTB, RAW), Toronto Western Hospital, Toronto, Ontario, Canada; Department of Neurosurgery (ARD), Geisinger Neurosciences Institute, Danville, Pennsylvania, U.S.A.

Received November 9, 2009. Final Revisions Submitted January 11, 2010. Correspondence to: Amir R. Dehdashti, Geisinger Neurosciences Institute, Department of Neurosurgery, 100 North Academy Avenue, Danville, 17822, Pennsylvania, U.S.A. 


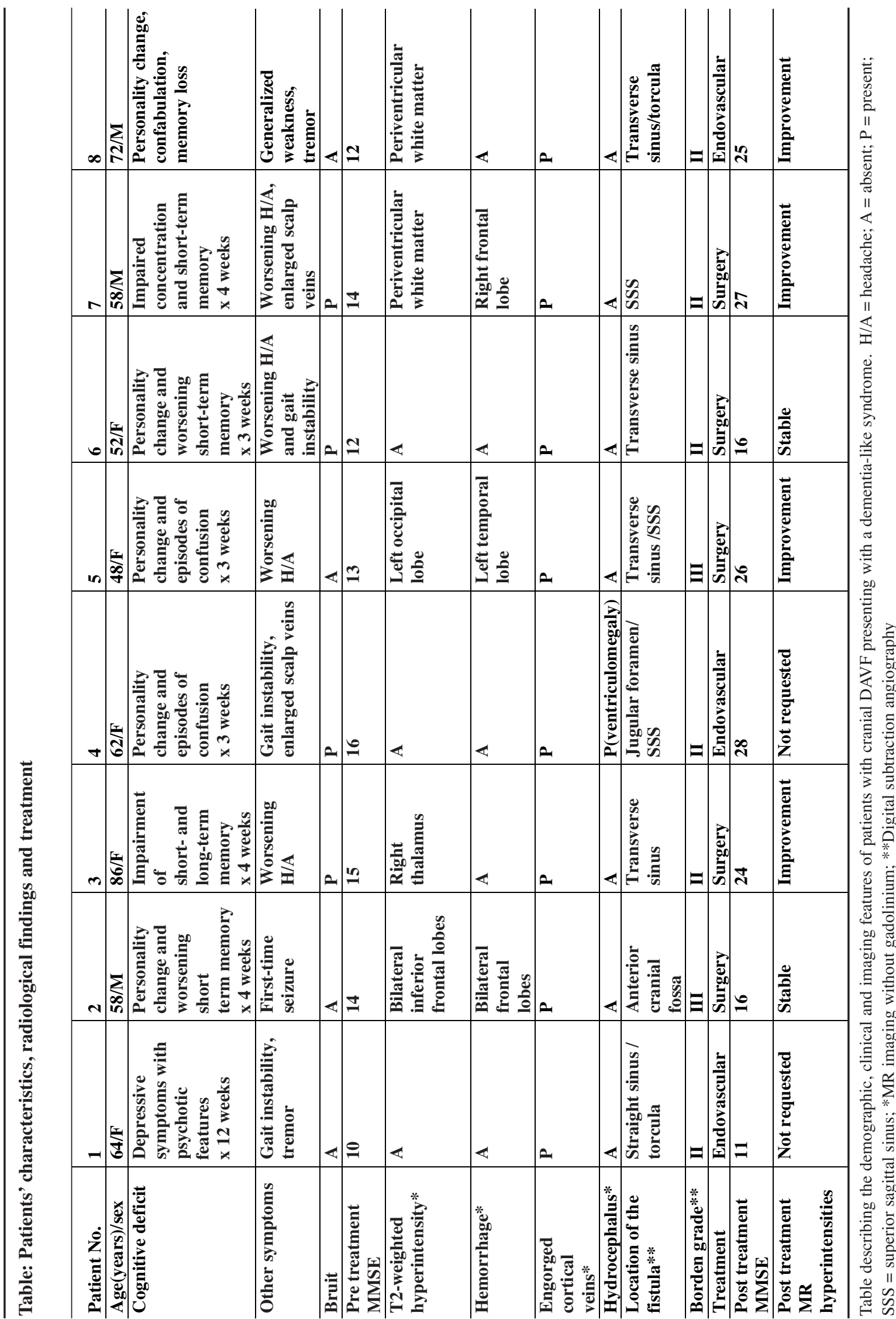



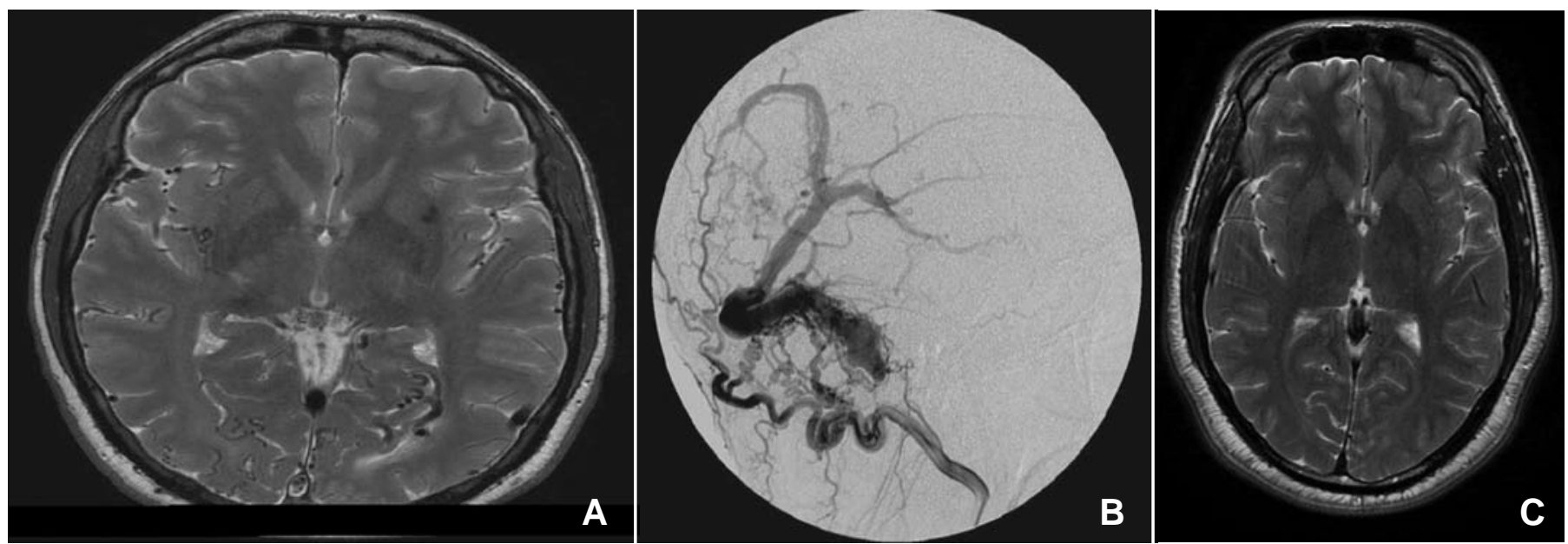

Figure: (A) Axial T2-weighted MRI demonstrating engorged venous channels over and within the cerebral hemispheres. (B) Conventional digital subtraction angiogram in the same patient demonstrating a Borden II DAVF of the transverse sinus with reflux into the straight sinus, deep venous system and cortical veins. (C) Post surgical disconnection of the fistula showing disappearance of the dilated veins and improvement of mild brain swelling.

\section{Discussion}

Progressive cognitive impairment as a result of venous congestive encephalopathy remains an under-recognized presentation of aggressive DAVF. In our series of 246 patients, eight patients $(3 \%)$ presented with predominantly progressive cognitive deficits in the absence of decreased level of consciousness or lateralizing findings. These patients tended to be younger than those most commonly afflicted with classical dementia syndromes and more rapidly progressive. Moreover, all demonstrated non-typical characteristics on history and physical examination that provoked MRI with specific sequences appropriate for diagnosis of these vascular abnormalities.

Magnetic resonance imaging depicted engorged venous channels on the surface of the cerebral and/or cerebellar hemispheres in all patients. ${ }^{4,6,7}$ Notably, in our series, T2weighted hyperintensities were apparent in five of eight patients. This is different from the series reported by Hurst et al, in which all patients had T2-weighted abnormalities. ${ }^{5}$ Digital subtraction angiograms confirmed the diagnosis of aggressive DAVF by documenting CVR in all cases. Engorged venous network and venous hypertension are the most likely causes of venous ischemia, congestive encephalopathy and MRI T2 changes. Hydrocephalus is not a prominent etiological factor for dementia in this group of patients. Prompt treatment of the fistula might alleviate symptomatic patients.

This study is limited by its retrospective nature. Furthermore one can argue that some dementia patients might have an unrelated DAVF. While the latter scenario is possible, one would not expect a clinical recovery in the majority of them after treatment and this has also been supported by others. ${ }^{89} \mathrm{We}$ conclude that atypical features on history and physical examination of especially younger patients with rapidly progressive dementia-like syndromes should provoke MRI. Physicians should consider DAVF as a rare but possible differential diagnosis of dementia especially in younger patients. A concerted effort should be made to document engorged venous channels over the cerebral and/or cerebellar hemispheres that may represent an early MRI clue to an underlying aggressive DAVF.

\section{REFERENCES}

1. Davies MA, TerBrugge K, Willinsky R, Coyne T, Saleh J, Wallace MC. The validity of classification for the clinical presentation of intracranial dural arteriovenous fistulas. J Neurosurg. 1996;85: 830-7.

2. van Dijk JM, terBrugge KG, Willinsky RA, Wallace MC. Clinical course of cranial dural arteriovenous fistulas with long-term persistent cortical venous reflux. Stroke. 2002;33:1233-6.

3. Lasjaunias P, Chiu M, ter Brugge K, Tolia A, Hurth M, Bernstein M. Neurological manifestations of intracranial dural arteriovenous malformations. J Neurosurg. 1986;64:724-30.

4. van Dijk JM, Willinsky RA. Venous congestive encephalopathy related to cranial dural arteriovenous fistulas. Neuroimaging Clin N Am. 2003;13:55-72.

5. Hurst RW, Bagley LJ, Galetta S, Glosser G, Lieberman AP, Trojanowski J, et al. Dementia resulting from dural arteriovenous fistulas: the pathologic findings of venous hypertensive encephalopathy. AJNR Am J Neuroradiol. 1998;19: 1267-73.

6. Willinsky R, Goyal M, terBrugge K, Montanera W. Tortuous, engorged pial veins in intracranial dural arteriovenous fistulas: correlations with presentation, location, and MR findings in 122 patients. AJNR Am J Neuroradiol. 1999;20:1031-6.

7. Willinsky R, Terbrugge K, Montanera W, Mikulis D, Wallace MC. Venous congestion: an MR finding in dural arteriovenous malformations with cortical venous drainage. AJNR Am J Neuroradiol. 1994;15:1501-7.

8. Kai Y, Ito K, Kinjo T, Hokama Y, Nagamine H, Kushi S, et al. Reversibility of cognitive disorder after treatment of dural arteriovenous fistulae. Neuroradiology. 2009;51:731-9.

9. Zeidman SM, Monsein LH, Arosarena O, Aletich V, Biafore JM, Dawson RC, et al. Reversibility of white matter changes and dementia after treatment of dural fistulas. AJNR Am J Neuroradiol. 1995;16:1080-3. 